\section{CASO CLÍNICO}

\title{
Reconstrucción total de columela con rinoplastia estética simultánea
}

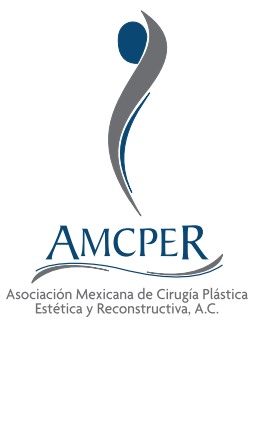

\author{
Dr. Ignacio Trigos Micoló*
}

Palabras clave: Reconstrucción de columela, narinas, rinoplastia estética.

Keywords: Reconstruction of columella, nostrils, aesthetic rhinoplasty.

\footnotetext{
* Cirujano Plástico Certificado en práctica privada. Académico Emérito de la Academia Mexicana de Cirugía.

El autor de este artículo no tienen conflicto de intereses qué declarar.

Recibido:

06 noviembre 2017 Aceptado para publicar: 01 octubre 2018
}

\section{RESUMEN}

La columela es una subunidad de la nariz de gran valor estético y funcional y puede ser afectada por variadas condiciones de tipo congénito o adquirido como infecciones, trauma, tumores o iatrogénico que hacen que su posible reconstrucción sea complicada. Se han descrito varias técnicas para lograrlo, que van desde el colgajo indio o frontal hasta diversos colgajos locales del piso nasolabial, de surcos nasogenianos, paredes laterales de la nariz, alas nasales, hasta injertos compuestos de diferentes áreas de la oreja, con o sin injertos de cartílago. Se presenta un caso de iatrogenia consecutiva a una rinoplastia convencional incorrecta, en la que, además de reconstruir toda la columela, se efectuó una rinoplastia correctiva simultáneamente. Discutimos las opciones reconstructivas haciendo énfasis en la conveniencia de esta variante, utilizando ambas narinas como sitio donador del colgajo, así como detalles técnicos que son útiles para lograr una mejor reconstrucción.

\section{ABSTRACT}

The columella is a sub-unit of the nose of great aesthetic and functional value which can be affected by several congenital or acquired conditions such as infections, trauma, tumors or iatrogenic injury that makes its possible reconstruction complicated. Several techniques have been described to achieve this, ranging from the Indian or frontal flap to various local flaps of the nasolabial floor, nasolabial folds, lateral walls of the nose, nasal wings, to grafts composed of different areas of the ear, with or without cartilage grafts. A case of iatrogenicity following an incorrect conventional rhinoplasty is presented, in which, in addition to reconstructing the entire columella, a corrective rhinoplasty was performed simultaneously. We discuss the reconstructive options emphasizing the convenience of this variant, using both nostrils as a donor site for the flap as well as technical details that are useful to achieve a better reconstruction.

\section{INTRODUCCIÓN}

L a columela es una subunidad anatómica de la nariz que se localiza en la línea media por debajo de la punta nasal, que divide ambas narinas y, de perfil, forma el ángulo nasolabial. Es un elemento de gran valor estético e influye directamente en la proyección de la punta de la nariz.

La columela es el asiento de patología diversa de origen diferente, que puede ser una alteración congénita desde ausencia parcial hasta total, como en los casos de fisuras del paladar primario o deformaciones propias de su desarrollo como las variantes de columela retraída o colgante. El segundo grupo está integrado por alteraciones adquiridas de diferente origen como traumático, tumoral, por aspiración de cocaína o iatrogénico, como necrosis por uso de sondas nasogástricas o postquirúrgicas. La etiopatogenia tan diversa y lo complicado de su anatomía hacen que su reconstrucción sea compleja y altamente especializada. ${ }^{1-3}$

Existen numerosas y variadas técnicas reconstructivas descritas en la literatura internacional desde hace varios siglos, como el colgajo indio de la región frontal, que ha sufrido innumerables modificaciones recientes, ${ }^{4-6}$ o la clásica obra de Tagliacozzi. ${ }^{7}$ Otras técnicas quirúrgicas fueron revisadas en los reportes de Kazanjian ${ }^{4}$ o Paletta, ${ }^{8}$ que incluyen variantes de todo tipo como injertos compuestos ${ }^{9}$ o diferentes diseños de colgajos locales. ${ }^{10-20}$

\section{CASO CLÍNICO}

Mujer de 28 años de edad que consultó a un cirujano solicitando una rinoplastia estética (Figura 1). La cirugía fue programada y se efectuó con un resultado desfavorable con 

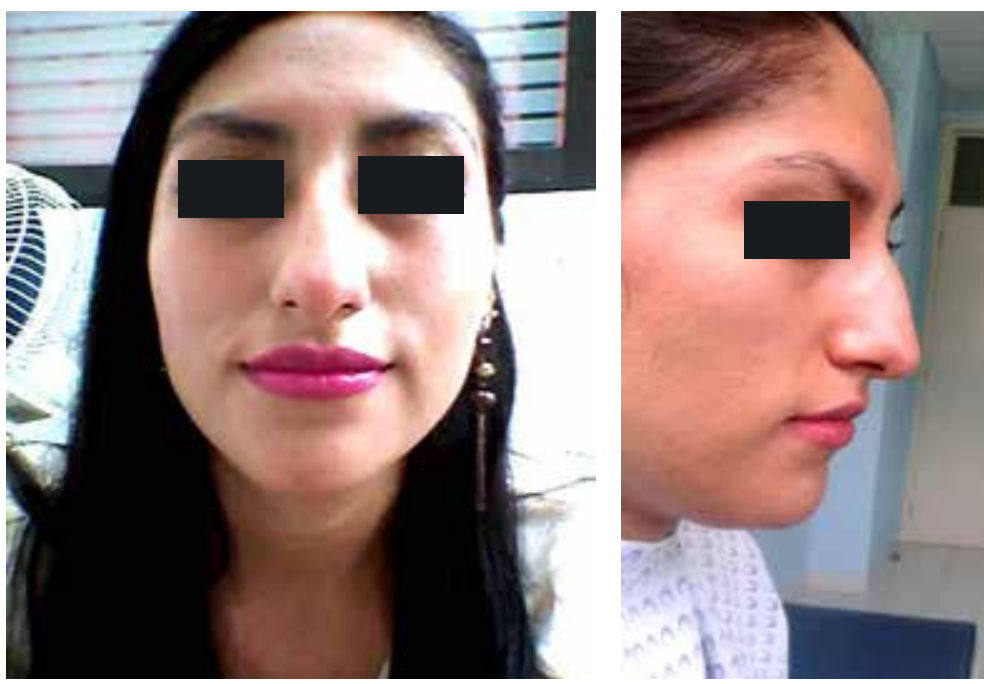

Figura 1. Preoperatorio de rinoplastia estética. necrosis total de columela. En esas condiciones acudió a mi consulta, que al revisarla planteaba un reto para la reconstrucción de la columela y deformación secundaria debida a la cirugía previa en una paciente resentida por tal evento y alterada emocional y económicamente (Figura 2).

A final de la valoración y estudio y debido al exceso de tejido disponible en el área de las narinas, decidí intentar la reconstrucción con ese tejido local, además de una rinoplastia secundaria simultánea con el siguiente plan quirúrgico: reconstrucción de columela con colgajos marginales de ambas narinas (Figura 3), complementadas con una rinoplastia secundaria (Figura 4).

El resultado inmediato fue satisfactorio en los objetivos trazados para la reconstrucción

Figura 2.

Postoperatorio con mal resultado, con

asimetrías, giba residual y necrosis de columela. Condiciones en las que se recibió y valoró por primera vez.
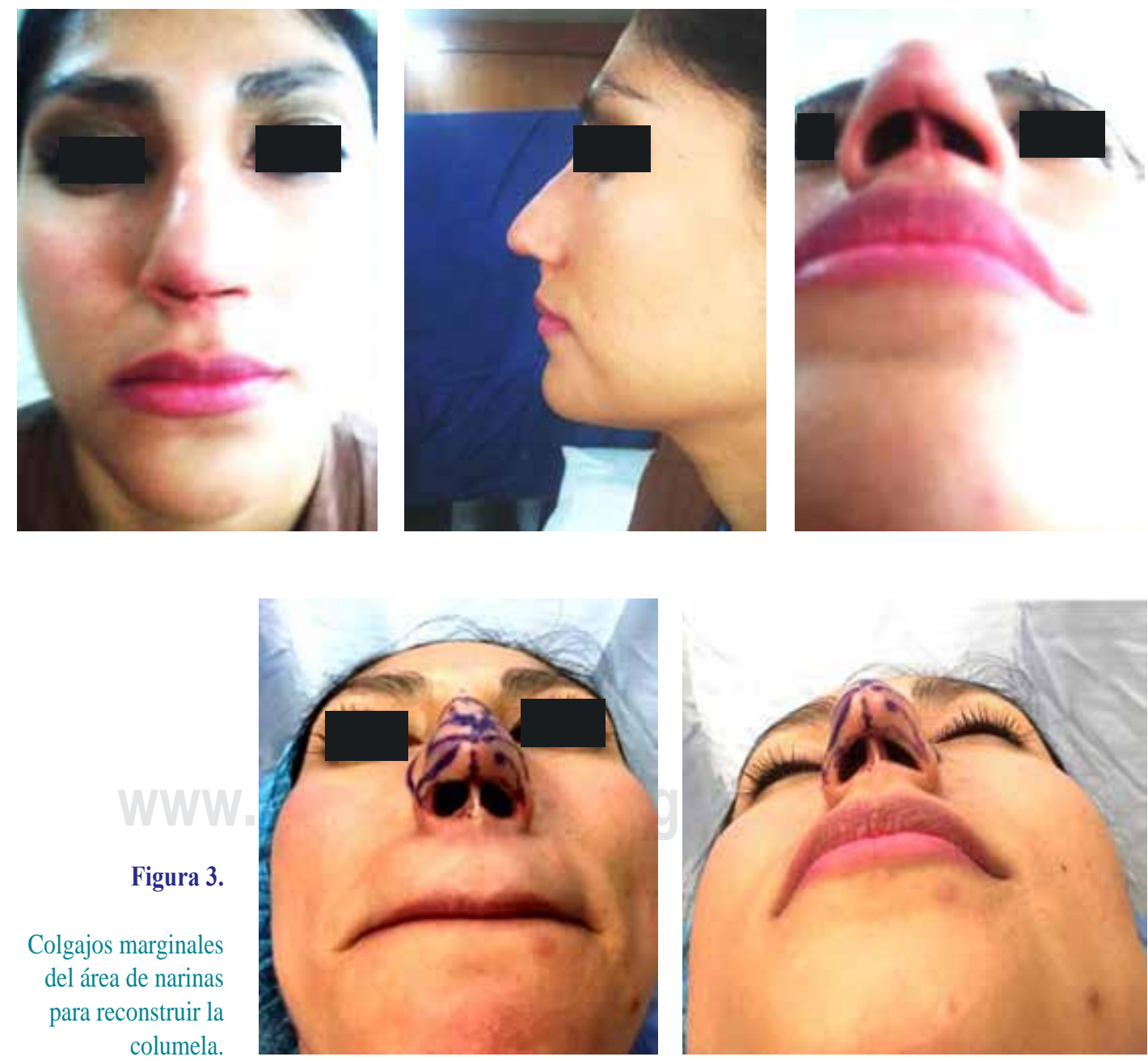
de columela con tejido similar y suficiente en cantidad, calidad y volumen, sin deformación en el área donadora. Con la rinoplastia se obtuvo una nueva forma nasal adecuada tanto de frente como de perfil; sin embargo, como secuela de la reconstrucción encontramos una muesca bilateral en el sitio de rotación de los colgajos y una discreta asimetría en la altura de las narinas (Figura 5).

Tres meses después de la intervención quirúrgica, decidimos, conjuntamente con anestesia local, corregir los detalles de las muescas en ambas narinas y proporcionar más proyección al ángulo nasolabial en el perfil izquierdo, obteniendo al mes de esta pequeña intervención un buen resultado final (Figura 6).

\section{DISCUSIÓN}

De las múltiples opciones reconstructivas de columela que están reportadas, debido a las características particulares de este caso, decidimos utilizar la variante reconstructiva descrita en este trabajo, manifestando no conocer previamente algún reporte similar, sólo con experiencias previas con la técnica descrita por Straith ${ }^{21}$ para pacientes con secuelas en las narinas por deformidades del paladar primario y por ello consideramos viable esta opción reconstructiva. Creíamos sinceramente haber encontrado una opción más para la solución de este problema, pero, revisando profundamente la bibliografía exis-

Figura 4.

Plan preoperatorio para rinoplastia estética secundaria simultánea.
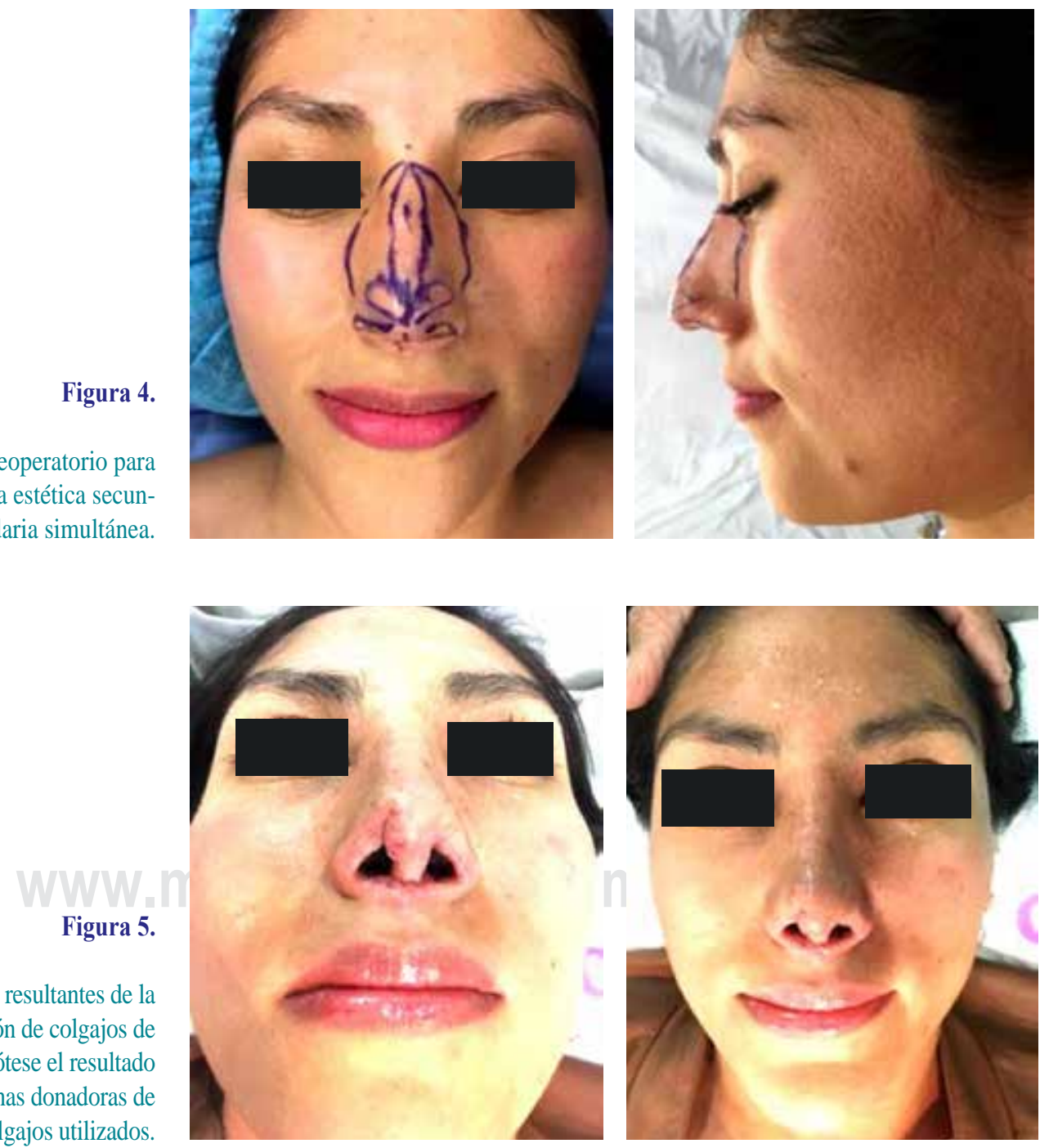

Muescas resultantes de la rotación de colgajos de narinas. Nótese el resultado de las zonas donadoras de los colgajos utilizados. 
Figura 6.

Resultado final a un mes de las correcciones realizadas.
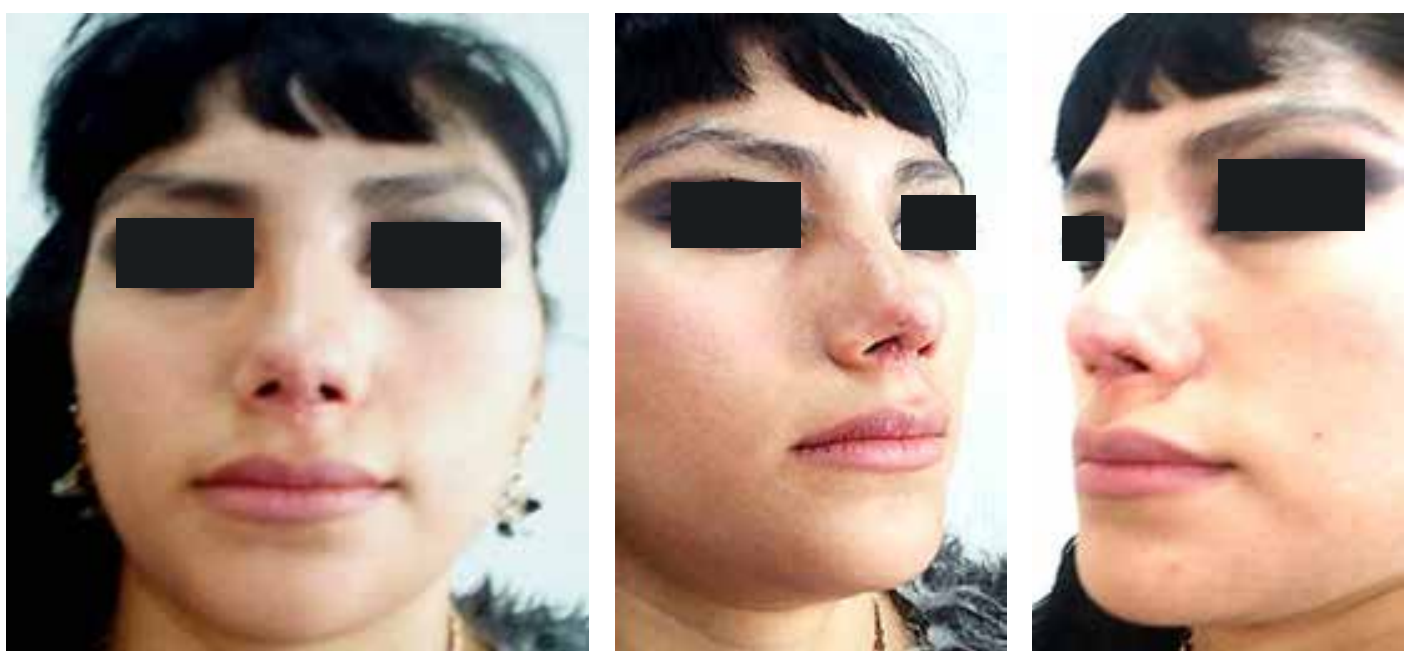

tente, encontramos algo idéntico descrito por Elbaz desde 1971.22-25

Para la utilización de la técnica descrita, se debe considerar la necesidad de retoques en las muescas secundarias a la rotación de los colgajos con pequeñas Z-plastias asimétricas que mejoren la continuidad de las narinas, aunque podemos recomendar, por la calidad circulatoria local del tejido utilizado, valorar la opción de efectuarlas primariamente en el primer tiempo quirúrgico.

\section{CONCLUSIONES}

La técnica descrita (Elbaz) es una buena opción reconstructiva para columela. Las secuelas de la zona donadora son mínimas con cicatrices imperceptibles con el tiempo. La combinación con una rinoplastia estética es factible, como demostramos en este caso.

\section{REFERENCIAS}

1. Margulis A, Icekson M, Billig A, Armon N, Neuman R, Adler N. Practical classification of columellar defects and surgical algorithm for their treatment. J Plast Reconstr Aesthet Surg 2013; 66: 577-579.

2. Ayhan M, Sevin A, Aytug Z, Gorgu M, Erdogan B. Reconstruction of congenital and acquired columellar defects: clinical review of 38 patients. J Craniofac Surg 2007; 18: 1500-1503.

3. Dolan R, Arena S. Reconstruction of the total columellar defect. Laryngoscope 1995; 105 (10):11411143.

4. Kazanjian $\vee \mathrm{H}$. The repair of nasal defects with the medial forehead flap. Surg Gynecol Obstet 1946; 83: 37-49.
5. Motamed S, Kalantar-Hormozi A J. Columella reconstruction with the Washio flap. Br J Plast Surg 2003; 56: 829-831.

6. Vulovíc D, Stepic N, Pavlovíc A, Milievic S, Piscevic B. Reconstruction of the columella and the tip of the nose with an island-shaped forehead flap. Vojnosanit Pregl 2011; 68 (3): 277-280.

7. Tagliacozzi G. De curtorum chirugia per institionem. Gaspare Bindoni the Younger, Venice. 1597

8. Paletta FX, Van Norman RT. Total reconstruction of the columella. Plast Reconstr Surg Transplant Bull 1962; 30: 322-328.

9. Kim YO, Park BY, Lee WJ. Aesthetic reconstruction of the nasal tip using a folded composite graft from the ear. Br J Plast Surg 2004; 57: 238-244.

10. Stricker M. Les mutilations de la columelle. Rev Stomatol 1996; 73: 485-494.

11. Sherris DA, Fuerstenberg J, Danahey D, Hilger PA. Reconstruction of the nasal columella. Arch Facial Plast Surg 2002; 4: 42-46.

12. Lunatschek C, Schwipper V, Scheithauer M. Soft tissue reconstruction of the nose. Facial Plast Surg 2011; 27: 249-257.

13. Jung DH, Lansangan LJ, Choi JM, Jang TY, Lee JJ. Subnasal flap for correction of columellar deformity. Plast Reconstr Surg 2007; 119: 885-890.

14. Lazaridis N. Unilateral subcutaneous pedicled nasolabial island flap for anterior mouth floor reconstruction. J Oral Maxillofac Surg 2003; 61: 182-190.

15. Lazaridis N, Zouloumis L, Venetis G, Karakasis D. The inferiorly and superiorly based nasolabial flap for the reconstruction of moderate-sized oronasal defects. J Oral Maxillofac Surg 1998; 56: 1255-1259.

16. Ozkus I, Cek DI, Ozkus K. The use of bifid nasolabial flaps in the reconstruction of the nose and columella. Ann Plast Surg 1992; 29: 461-463.

17. Yanai A, Nagata S, Tanaka H. Reconstruction of the columella with bilateral nasolabial flaps. Plast Reconstr Surg 1986; 77: 129-132.

18. Trimarchi MM, Bussi M, Sinico RA, Meroni P, Specks $U$. Cocaine-induced midline destructive lesions. An 
autoimmune disease? Autoimmun Rev 2013; 12: 496-500.

19. Guyuron B, Afrooz PN. Correction of cocaine-related nasal defects. Plast Reconstr Surg 2008; 121: 10151023.

20. Barton FE. Jr. Aesthetic aspects of nasal reconstruction. Clin Plast Surg 1988; 15: 155-166.

21. Straith CL, Straith RE, Lawson JM. Reconstruction of the harelip nose. Plast Reconstr Surg 1957; 20: 455-459.

22. Ndiaye L, Sankale A, Ndiaye A, Foba L. Columella reconstruction with Elbaz's flap: a case report and literature review. Internet J Plast Surg 2016; 12: 1-4.

23. Elbaz JS. Columella repair by a simple 1 -stage procedure (apropos of a case seen more than 4 years later). Ann Chir Plast 1971; 16: 25-29.
24. Saad MN, Barron JN. Reconstruction of the columella with alar margin flaps. Br J Plast Surg 1980; 33: 427-429.

25. Demir Z, Ozdil K, Karamüsel S, Yüce S, Oktem F, Celebioğlu S. Reconstruction of the columella with bilateral nostril sill flaps. Ann Otol Rhinol Laryngol 2006; 115: 239-242.

Correspondencia:

Dr. Ignacio Trigos Micoló

Durango Núm. 33-2, Col. Roma,

Alcaldía Cuauhtémoc, 06700,

Ciudad de México, México.

Tels. (55) 55110444,

E-mail: dritrigos@live.com.mx 